\title{
Check de votre prévoyance professionnelle (LPP)
}

Profitez-vous de tous les avantages des solutions de prévoyance pour médecins?

Prenez cinq minutes pour le check de votre prévoyance professionnelle LPP et regardez si vous disposez d'un potentiel d'optimalisation de votre prévoyance professionnelle:

Connaissez-vous les solutions des caisses de pension de FMH Insurance Services?

$\bigcirc$ oui $\bigcirc$ non

Nos offres sont orientées sur les besoins des médecins. Selon l'offre vous profitez p.ex. des coûts d'administration peu élevés ou d'un taux de conversion élevé. Une comparaison d'optimalisation vaut la peine, car votre contrat actuel peut normalement être résilié au 30 juin pour la fin de l'année.

N'avez-vous plus de possibilités de rachat dans votre LPP?

○ oui $\bigcirc$ non

Les rachats LPP peuvent être entièrement déduits de votre revenu imposable. Ainsi vous pouvez selon votre montant imposable et votre lieu du domicile économiser jusqu'à 45\% d'impôts. Les possibilités de rachats diffèrent d'une caisse à l'autre.

Connaissez-vous les prestations de votre LPP et correspondent-elles à vos besoins?

$\bigcirc$ oui $\bigcirc$ non

Seul celui qui connaît parfaitement sa caisse de pension peut planifier sa retraite d'une manière optimale. Les conseillers de FMH Insurance Services vous conseillent volontiers et vous montrent quelles prestations doivent être assurées pour vous éviter des lacunes de couverture.

Avez-vous observé si vous pouviez augmenter la cotisation d'épargne?

o oui $\bigcirc$ non Lors de l'augmentation de la cotisation d'épargne du plan LPP vous disposez d'un montant de rachat potentiel plus haut, solution à utiliser pour une optimalisation fiscale. Une caisse de pension corporative permet de trouver une solution pour le paiement d'une cotisation d'épargne atteignant jusqu'à 25\% du salaire assuré.

Votre épouse/votre époux est-elle/il affiliée/é à une prévoyance professionnelle? O oui non Souvent dans la réalité le conjoint travaillant au cabinet ne reçoit pas de salaire pour son activité et ainsi n'est pas affilié à une prévoyance LPP. En versant un salaire, le conjoint peut participer à une prévoyance LPP et en plus souscrire un $3^{\text {ème }}$ pilier lié $a$.

Est-il nécessaire de transformer tout votre avoir de vieillesse en rente?

o oui $\bigcirc$ non

En séparant la partie obligatoire de la partie surobligatoire de la prévoyance vous augmentez votre flexibilité. A côté des avantages fiscaux vous pouvez après votre retraite disposer à votre convenance de votre argent, tout en maintenant vos besoins de base par le versement d’une rente assurée, prélevée sur la partie obligatoire de votre prévoyance.

Evaluation

$0 \times$ non: $\quad$ Sincères félicitations, vous avez déjà envisagé une optimalisation de votre prévoyance

1-6×non: Vous disposez d'un potentiel d'optimalisation important. Nous vous conseillons de vous adresser rapidement à un spécialiste en prévoyance de FMH Insurance Services.

\section{Talon-réponse}

Prière de l'envoyer ou le faxer au: 0319595010

Prénom / Nom

Adresse

NPA / Lieu

Date de naissance

Téléphone privé / cabinet

Atteignable le plus facilement (heure)

Adresse e-mail

Je désire un conseil personnalisé. Prière de $\mathrm{m}^{\prime}$ appeler.

Je suis intéressé à:
O Pilier 3a
O Planification retraite
Caisse de pension LPP
P Planification financière
O Assurance protection juridique
O Assurance RC professionnel 